\title{
A Necessary and Sufficient Condition for Pseudo-symmetric Positive Solutions of Boundary Value Problems
}

\author{
Yan $\mathrm{Luo}^{1}$ \\ ${ }^{1}$ Department of Mathematics, Hunan University of Science and Technology, Xiangtan, Hunan 411201, China \\ Correspondence: Yan Luo, Department of Mathematics, Hunan University of Science and Technology, Xiangtan, \\ Hunan 411201, China. E-mail: luoyan2527@126.com
}

Received: October 23, 2014 Accepted: November 8, 2014 Online Published: November 21, 2014

doi:10.5539/jmr.v6n4p149 URL: http://dx.doi.org/10.5539/jmr.v6n4p149

The research supported by the Scientific Research Fund of Hunan Provincial Education Department (13C319)

\begin{abstract}
We apply the monotone iterative technique to the second-order boundary value problems. We obtain a necessary and sufficient condition and discuss the uniqueness, a iterative sequence and an error estimation for pseudosymmetric positive solutions. Moreover, an example is given to illustrate the applicability of our results.
\end{abstract}

Keywords: boundary value problems, pseudo-symmetric positive solutions, monotone iterative technique, necessary and sufficient condition

\section{Introduction}

Consider the pseudo-symmetric boundary value problem

$$
\left\{\begin{array}{l}
u^{\prime \prime}(t)+f(t, u(t))=0, t \in(0,1) \\
u(0)=0, u(\eta)=u(1)
\end{array}\right.
$$

where $\eta \in(0,1)$. For $\eta \in(0,1)$, a function $u \in C[0,1]$ is said to be pseudo-symmetric if $u$ is symmetric over the interval $[\eta, 1]$. That is, $u(t)=u(1+\eta-t), t \in[\eta, 1]$. By a pseudo-symmetric positive solution of (1.1), we mean a pseudo-symmetric function $u \in C^{2}[0,1]$ such that $u(t)>0$ for $t \in(0,1)$, and $u(t)$ satisfies (1.1).

Recently, many authors have focused on the question of symmetric positive solutions for ordinary differential equation boundary value problems, for example, see (Avery \& Henderson, 2000; Çetin \& Topal, 2012; Graef \& Kong, 2008; Hamal \& Yoruk, 2010; Jiang, Liu \& Wu, 2013; Luo \& Luo, 2010; Luo \& Luo, 2012; Lin \& Zhao, 2013; Tersenov, 2014) and the references therein. In (Avery \& Henderson, 2003), Avery and Henderson gave the definition of pseudo-symmetric function. Since then, some papers have discussed the pseudo-symmetric question and established sufficient conditions for the existence of pseudo-symmetric positive solutions, see (Feng, Zhang \& Ge, 2010; Guo, Han \& Chen, 2010; Ji, 2008; Ma \& Ge, 2007; Pang, 2009; Sun \& Zhao, 2014 ). To the best of the authors' knowledge there is little known about necessary and sufficient conditions for second-order pseudosymmetric nonlinear boundary value problem. Motivated by the works mentioned above, we aim to establish a necessary and sufficient condition for the existence of pseudo-symmetric positive solution of (1.1) by applying the monotone iterative technique.

The organization of the paper is as follows. Section 2 contains some preliminary lemmas and the basic assumptions. In Section 3, by applying the monotone iterative technique, we obtain a sufficient and necessary condition for the existence of at least one pseudo-symmetric positive solution for problem (1.1), we also discuss the uniqueness, a iterative sequence and an error estimation for the pseudo-symmetric positive solution to (1.1). In Section 4, an example will be presented to illustrate the applicability of our results.

\section{Preliminaries}

By routine calculations we have the following result. 
Lemma 2.1. Let $v \in C[0,1]$, then the boundary value problem

$$
\left\{\begin{array}{l}
u^{\prime \prime}(t)+v(t)=0, t \in(0,1), \\
u(0)=0, u(\eta)=u(1)
\end{array}\right.
$$

has a unique solution

$$
u(t)= \begin{cases}\int_{0}^{t} \int_{s}^{\frac{1+\eta}{2}} v(r) d r d s, & 0 \leq t \leq \frac{1+\eta}{2}, \\ \int_{0}^{\eta} \int_{s}^{\frac{1+\eta}{2}} v(r) d r d s+\int_{t}^{1} \int_{\frac{1+\eta}{2}}^{s} v(r) d r d s, & \frac{1+\eta}{2} \leq t \leq 1,\end{cases}
$$

where $\eta \in(0,1)$.

Lemma 2.2. Assume that $u(t)$ is a pseudo-symmetric positive solution of (1.1). Then there exist constants $c_{1}$, $c_{2}$ with $0<c_{1}<1<c_{2}$ such that

$$
c_{1} \omega(t) \leq u(t) \leq c_{2} \omega(t), t \in[0,1]
$$

Proof. To prove (2.1) holds, first note that $u(t)>0$ and $u^{\prime \prime}(t) \leq 0$ for $t \in(0,1)$. From the pseudo-symmetry of $u(t)$, we have $u\left(\frac{1+\eta}{2}\right)=\max _{t \in[0,1]} u(t)$. If $t \in\left(0, \frac{1+\eta}{2}\right)$, then $u(t) \geq \frac{2 u\left(\frac{1+\eta}{2}\right)}{1+\eta} t$, and if $t \in\left(\frac{1+\eta}{2}, 1\right)$, then $u(t) \geq \frac{2 u\left(\frac{1+\eta}{2}\right)}{1+\eta}(1+\eta-t)$. Hence, for $t \in(0,1), u(t) \geq \frac{2 u\left(\frac{1+\eta}{2}\right)}{1+\eta} \omega(t)$. Thus, we take $c_{1}$ with $0<c_{1}<\min \left\{1, \frac{2 u\left(\frac{1+\eta}{2}\right)}{1+\eta}\right\}$. The proof of the other half of (2.1) is similar, we have that $u(t) \leq u^{\prime}(0) \omega(t)$, thus, choosing $c_{2}>\max \left\{1, u^{\prime}(0)\right\}$. The proof is complete.

Throughout this paper, we assume the following conditions hold without further mention.

(H1) $f:(0,1) \times[0, \infty) \rightarrow[0, \infty)$ is continuous. For $(t, u) \in(0,1) \times[0, \infty), \eta \in(0,1), f(t, u)$ is pseudo-symmetric in $t$, i.e., $f$ satisfies

$$
f(1+\eta-t, u)=f(t, u), t \in[\eta, 1] .
$$

(H2) For $(t, u) \in(0,1) \times[0, \infty), f(t, u)$ is nondecreasing in $u$ and there exists a constant $\lambda \in(0,1)$ such that if $\sigma \in(0,1]$, then

$$
\sigma^{\lambda} f(t, u) \leq f(t, \sigma u)
$$

Example 2.1. Study the equation

$$
f(t, u)=|2 t-\eta-1| u^{\frac{1}{4}},(t, u) \in(0,1) \times[0, \infty) .
$$

It is easy to see that the function $f$ satisfies assumptions $(\mathrm{H} 1)$ and $(\mathrm{H} 2)$. In fact, if $\sigma \in(0,1]$, there exists constant $\lambda$ with $\frac{1}{4} \leq \lambda<1$ such that $f(t, \sigma u) \geq \sigma^{\lambda} f(t, u)$.

Remark 2.1. Expression (2.2) implies that if $\sigma \in[1, \infty)$, we have

$$
f(t, \sigma u) \leq \sigma^{\lambda} f(t, u)
$$

For convenience, let

$$
\omega(t)=\min \{t, 1+\eta-t\}, t \in[0,1],
$$

and $E$ be the Banach space $C^{2}[0,1]$, and define

$$
\begin{gathered}
P=\left\{\begin{array}{c}
u \in E: u(0)=0, u(t)>0 \text { for } t \in(0,1), u(t)=u(1+\eta-t) \text { for } \\
t \in[\eta, 1] \text { and there exist constants } l_{u}, L_{u} \text { with } 0<l_{u}<1<L_{u}
\end{array}\right. \\
\text { such that } \left.l_{u} \omega(t) \leq u(t) \leq L_{u} \omega(t) \text { for } t \in[0,1]\right\} .
\end{gathered}
$$

Remark 2.2. The set $P$ is not a cone as it is not closed. 


\section{Main Results}

Theorem 3.1. Assume (H1) and (H2) hold. Then the boundary value problem (1.1) has at least one pseudosymmetric positive solution if and only if

$$
0<\int_{0}^{1} f(t, \omega(t)) d t<\infty
$$

Proof. Necessity. Suppose first that $u(t)$ is a pseudo-symmetric positive solution of (1.1), we will show that (3.1) holds.

Let $c_{1}$ and $c_{2}$ be given as in Lemma 2.2 for this $u(t)$. By Lemma 2.2, $u(t)$ satisfies (2.1). Hence, by (H2), Remark (2.1) and (2.1), we have

$$
\begin{aligned}
\int_{0}^{1} f(t, \omega(t)) d t & \leq \int_{0}^{1} f\left(t, c_{1}^{-1} u(t)\right) d t \\
& \leq c_{1}^{-\lambda} \int_{0}^{1} f(t, u(t)) d t \\
& =c_{1}^{-\lambda}\left[u^{\prime}(0)-u^{\prime}(1)\right]<\infty
\end{aligned}
$$

and

$$
\begin{aligned}
\int_{0}^{1} f(t, \omega(t)) d t & \geq \int_{0}^{1} f\left(t, c_{2}^{-1} u(t)\right) d t \\
& \geq c_{2}^{-\lambda} \int_{0}^{1} f(t, u(t)) d t \\
& =c_{2}^{-\lambda}\left[u^{\prime}(0)-u^{\prime}(1)\right]>0 .
\end{aligned}
$$

Now, (3.1) follows from (3.2) and (3.3).

Sufficiency. Now assume that (3.1) holds, we will show that (1.1) has at least one pseudo-symmetric positive solution.

Define the operator $T: E \rightarrow E$ by

$$
T u(t)= \begin{cases}\int_{0}^{t} \int_{s}^{\frac{1+\eta}{2}} f(r, u(r)) d r d s, & 0 \leq t \leq \frac{1+\eta}{2}, \\ \int_{0}^{\eta} \int_{s}^{\frac{1+\eta}{2}} f(r, u(r)) d r d s+\int_{t}^{1} \int_{\frac{1+\eta}{2}}^{s} f(r, u(r)) d r d s, & \frac{1+\eta}{2} \leq t \leq 1 .\end{cases}
$$

It is clear that $u$ is a solution of (1.1) if and only if $u$ is a fixed point of $T$.

Claim 1. The operator $T: P \rightarrow P$ is completely continuous and nondecreasing.

We first note that for $u \in P$ we have $T u(0)=0, T u(t)>0$ for $t \in(0,1)$. We now prove that $T u$ is pseudo-symmetric about $\eta \in(0,1)$.

In fact, for $t \in\left[\eta, \frac{1+\eta}{2}\right]$, then $1+\eta-t \in\left[\frac{1+\eta}{2}, 1\right]$. From (H1), we have

$$
\begin{aligned}
T u(1+\eta-t) & =\int_{0}^{\eta} \int_{s}^{\frac{1+\eta}{2}} f(r, u(r)) d r d s+\int_{1+\eta-t}^{1} \int_{\frac{1+\eta}{2}}^{s} f(r, u(r)) d r d s \\
& =\int_{0}^{\eta} \int_{s}^{\frac{1+\eta}{2}} f(r, u(r)) d r d s+\int_{\eta}^{t} \int_{\frac{1+\eta}{2}}^{1+\eta-s} f(r, u(r)) d r d s \\
& =\int_{0}^{t} \int_{s}^{\frac{1+\eta}{2}} f(r, u(r)) d r d s+\int_{\eta}^{t} \int_{s}^{\frac{1+\eta}{2}} f(r, u(r)) d r d s \\
& =\int_{0}^{t} \int_{s}^{\frac{1+\eta}{2}} f(r, u(r)) d r d s=T u(t)
\end{aligned}
$$


and for $t \in\left[\frac{1+\eta}{2}, 1\right]$, we note that $1+\eta-t \in\left[\eta, \frac{1+\eta}{2}\right]$. Thus,

$$
\begin{aligned}
T u(1+\eta-t) & =\int_{0}^{1+\eta-t} \int_{s}^{\frac{1+\eta}{2}} f(r, u(r)) d r d s \\
& =\int_{0}^{\eta} \int_{s}^{\frac{1+\eta}{2}} f(r, u(r)) d r d s+\int_{\eta}^{1+\eta-t} \int_{s}^{\frac{1+\eta}{2}} f(r, u(r)) d r d s \\
& =\int_{0}^{\eta} \int_{s}^{\frac{1+\eta}{2}} f(r, u(r)) d r d s+\int_{t}^{1} \int_{1+\eta-s}^{\frac{1+\eta}{2}} f(r, u(r)) d r d s \\
& =\int_{0}^{\eta} \int_{s}^{\frac{1+\eta}{2}} f(r, u(r)) d r d s+\int_{t}^{1} \int_{\frac{1+\eta}{2}}^{s} f(r, u(r)) d r d s=T u(t) .
\end{aligned}
$$

For $t \in\left[0, \frac{1+\eta}{2}\right]$, we have $\omega(t)=\min \{t, 1+\eta-t\}=t$, so for any $u \in P$, from (2.2), (2.3) and (3.1),

$$
\begin{aligned}
T u(t) & =\int_{0}^{t} \int_{s}^{\frac{1+\eta}{2}} f(r, u(r)) d r d s \\
& \leq \int_{0}^{t} \int_{0}^{1} f\left(r, L_{u} \omega(r)\right) d r d s \\
& \leq t L_{u}^{\lambda} \int_{0}^{1} f(r, \omega(r)) d r \leq L_{T u} \omega(t)
\end{aligned}
$$

and for $t \in\left[\frac{1+\eta}{2}, 1\right]$, we have $\omega(t)=1+\eta-t$,

$$
\begin{aligned}
T u(t) & =\int_{0}^{\eta} \int_{s}^{\frac{1+\eta}{2}} f(r, u(r)) d r d s+\int_{t}^{1} \int_{\frac{1+\eta}{2}}^{s} f(r, u(r)) d r d s \\
& \leq \int_{0}^{\eta} \int_{0}^{1} f\left(r, L_{u} \omega(r)\right) d r d s+\int_{t}^{1} \int_{0}^{1} f\left(r, L_{u} \omega(r)\right) d r d s \\
& \leq(1+\eta-t) L_{u}^{\lambda} \int_{0}^{1} f(r, \omega(r)) d r \leq L_{T u} \omega(t),
\end{aligned}
$$

where $L_{T u}>\max \left\{1, L_{u}^{\lambda} \int_{0}^{1} f(r, \omega(r)) d r\right\}$.

On the other hand, for $t \in\left[0, \frac{1+\eta}{2}\right]$, there exists constant $\xi \in\left(0, \frac{1+\eta}{2}\right)$ such that

$$
\begin{aligned}
T u(t) & =\int_{0}^{t} \int_{s}^{\frac{1+\eta}{2}} f(r, u(r)) d r d s \\
& \geq \int_{0}^{t} \int_{\frac{\xi}{1+\eta}}^{\frac{1+\eta}{2}} f(r, u(r)) d r d s \\
& \geq t \int_{\xi}^{\frac{1+\eta}{2}} f\left(r, l_{u} \omega(r)\right) d r d s \\
& \geq t l_{u}^{\lambda} \int_{\xi}^{\frac{1+\eta}{2}} f(r, \omega(r)) d r \geq l_{T u} \omega(t)
\end{aligned}
$$

and for $t \in\left[\frac{1+\eta}{2}, 1\right]$, there exist constants $\zeta \in\left(\frac{1+\eta}{2}, 1\right)$ such that

$$
\int_{t}^{1} \int_{\frac{1+\eta}{2}}^{s} f(r, u(r)) d r d s \geq(1-t) \int_{\frac{1+\eta}{2}}^{\zeta} f(r, u(r)) d r,
$$


and $M>0$ such that $\min \left\{\int_{\eta}^{\frac{1+\eta}{2}} f(r, \omega(r)) d r, \int_{\frac{1+\eta}{2}}^{\zeta} f(r, \omega(r)) d r\right\}>M$. Thus,

$$
\begin{aligned}
T u(t) & =\int_{0}^{\eta} \int_{s}^{\frac{1+\eta}{2}} f(r, u(r)) d r d s+\int_{t}^{1} \int_{\frac{1+\eta}{2}}^{s} f(r, u(r)) d r d s \\
& \geq \eta \int_{\eta}^{\frac{1+\eta}{2}} f\left(r, l_{u} \omega(r)\right) d r+(1-t) \int_{\frac{1+\eta}{2}}^{\zeta} f\left(r, l_{u} \omega(r)\right) d r \\
& \geq l_{u}^{\lambda}\left[\eta \int_{\eta}^{\frac{1+\eta}{2}} f(r, \omega(r)) d r+(1-t) \int_{\frac{1+\eta}{2}}^{\zeta} f(r, \omega(r)) d r\right] \\
& \geq l_{u}^{\lambda} M(1+\eta-t) \geq l_{T u} \omega(t),
\end{aligned}
$$

where $0<l_{T u}<\min \left\{1, l_{u}^{\lambda} \int_{\xi}^{\frac{1+\eta}{2}} f(r, \omega(r)) d r, l_{u}^{\lambda} M\right\}$.

Hence, it follows from (3.5)-(3.8) that there exist constants $l_{T u}$ and $L_{T u}$ with $0<l_{T u}<1<L_{T u}$ such that

$$
l_{T u} \omega(t) \leq T u(t) \leq L_{T u} \omega(t) \text { for } t \in[0,1] .
$$

Consequently, $T u \in P$, and so $T: P \rightarrow P$. A standard argument can be used to show that $T$ is completely continuous. From (H2), it is easy to see that $T$ is nondecreasing for $u$. Hence, Claim 1 holds.

Claim 2. Let $\delta$ and $\gamma$ be fixed numbers satisfying

$$
0<\delta \leq l_{T \omega}^{1 /(1-\lambda)}, \gamma \geq L_{T \omega}^{1 /(1-\lambda)},
$$

and assume

$$
\begin{gathered}
u_{0}=\delta \omega(t), v_{0}=\gamma \omega(t), \\
u_{n}=T u_{n-1}, v_{n}=T v_{n-1}, n=1,2, \ldots .
\end{gathered}
$$

Then,

$$
u_{0} \leq u_{1} \leq \cdots \leq u_{n} \leq \cdots \leq v_{n} \leq \cdots \leq v_{1} \leq v_{0}
$$

and there exists $u^{*} \in P$ such that

$$
u_{n}(t) \rightarrow u^{*}(t), v_{n}(t) \rightarrow u^{*}(t), \text { uniformly on }[0,1]
$$

In fact, $0<l_{T \omega}<1<L_{T \omega}$ since $T \omega(t) \in P$. So, $0<\delta<1<\gamma$. From (3.10), we have $u_{0}, v_{0} \in P$ and $u_{0} \leq v_{0}$. On the other hand, for $t \in\left[0, \frac{1+\eta}{2}\right]$, from (2.2) and (2.3),

$$
\begin{aligned}
u_{1}=T u_{0}(t) & =\int_{0}^{t} \int_{s}^{\frac{1+\eta}{2}} f(r, \delta \omega(r)) d r d s \\
& \geq \delta^{\lambda} \int_{0}^{t} \int_{s}^{\frac{1+\eta}{2}} f(r, \omega(r)) d r d s \\
& =\delta^{\lambda} T \omega(t) \geq \delta^{\lambda} l_{T \omega} \omega(t) \\
& \geq \delta^{\lambda} \delta^{1-\lambda} \omega(t)=u_{0}
\end{aligned}
$$

and

$$
\begin{aligned}
v_{1}=T v_{0}(t) & =\int_{0}^{t} \int_{s}^{\frac{1+\eta}{2}} f(r, \gamma \omega(r)) d r d s \\
& \leq \gamma^{\lambda} T \omega(t) \leq \gamma^{\lambda} L_{T \omega} \omega(t) \\
& \leq \gamma^{\lambda} \gamma^{1-\lambda} \omega(t)=v_{0} .
\end{aligned}
$$

For $t \in\left[\frac{1+\eta}{2}, 1\right]$,

$$
\begin{aligned}
u_{1}=T u_{0}(t) & =\int_{0}^{\eta} \int_{s}^{\frac{1+\eta}{2}} f(r, \delta \omega(r)) d r d s+\int_{t}^{1} \int_{\frac{1+\eta}{2}}^{s} f(r, \delta \omega(r)) d r d s \\
& \geq \delta^{\lambda} T \omega(t) \geq \delta^{\lambda} l_{T \omega} \omega(t) \\
& \geq \delta^{\lambda} \delta^{1-\lambda} \omega(t)=u_{0}
\end{aligned}
$$


and

$$
\begin{aligned}
v_{1}=T v_{0}(t) & =\int_{0}^{\eta} \int_{s}^{\frac{1+\eta}{2}} f(r, \gamma \omega(r)) d r d s+\int_{t}^{1} \int_{\frac{1+\eta}{2}}^{s} f(r, \gamma \omega(r)) d r d s \\
& \leq \gamma^{\lambda} T \omega(t) \leq \gamma^{\lambda} L_{T \omega} \omega(t) \\
& \leq \gamma^{\lambda} \gamma^{1-\lambda} \omega(t)=v_{0} .
\end{aligned}
$$

From (3.14)-(3.17), we have that $u_{1} \geq u_{0}$ and $v_{1} \leq v_{0}$ for $t \in[0,1]$. Since $u_{0} \leq v_{0}$ and $T$ is nondecreasing, by induction, (3.12) holds.

Let $c_{0}=\frac{\delta}{\gamma}$, then $0<c_{0}<1$. It follows from

$$
T(c u) \geq c^{\lambda} T u, \text { if } 0<c<1, u \in P
$$

that for any natural number $n$

$$
u_{n}=T u_{n-1}=T^{n} u_{0}=T^{n}(\delta \omega(t))=T^{n}\left(c_{0} \gamma \omega(t)\right) \geq c_{0}^{\lambda^{n}} T^{n}(\gamma \omega(t))=c_{0}^{\lambda^{n}} v_{n}
$$

Thus, for each natural number $n$ an $p^{*}$, we have

$$
0 \leq u_{n+p^{*}}-u_{n} \leq v_{n}-u_{n} \leq\left(1-c_{0}^{\lambda^{n}}\right) v_{n} \leq\left(1-c_{0}^{\lambda^{n}}\right) \gamma \omega(t),
$$

which implies that there exists $u^{*} \in P$ such that (3.13) holds, and Claim 2 holds.

Let $n \rightarrow \infty$ in (3.11), we obtain $u^{*}(t)=T u^{*}(t)$, which is a pseudo-symmetric positive solution of (1.1). The proof of the theorem is now complete.

Theorem 3.2. Assume (H1), (H2) and (3.1) hold. Then

(i) (1.1) has a unique pseudo-symmetric positive solution $u^{*}(t)$, and there exist constants $l, L \in R$ with $0<l<1<L$ such that

$$
l \omega(t) \leq u^{*}(t) \leq L \omega(t), \quad t \in[0,1] .
$$

(ii) For any initial value $x_{0} \in P$, there exists a sequence $x_{n}(t)$ which uniformly converges to the unique pseudosymmetric positive solution $u^{*}(t)$ for (1.1), and we have the error estimation

$$
\max _{t \in[0,1]}\left|x_{n}(t)-u^{*}(t)\right|=O\left(1-k^{\lambda^{n}}\right),
$$

where $k$ is a constant with $0<k<1$ and determined by $x_{0}$.

Proof. Let $u_{0}, v_{0}, u_{n}, v_{n}$ be defined in (3.10) and (3.11).

(i) It follows from Theorem 3.1 that (1.1) has at least one pseudo-symmetric positive solution $u^{*}(t) \in P$, which implies that there exist constants $l$ and $L$ with $0<l<1<L$ such that $u^{*}(t)$ satisfies (3.19). Let $v^{*}(t)$ be another pseudo-symmetric positive solution of (1.1), then from Lemma 2.2 we have that there exist constants $c_{1}$ and $c_{2}$ with $0<c_{1}<1<c_{2}$ such that

$$
c_{1} \omega(t) \leq v^{*}(t) \leq c_{2} \omega(t) \text { for } t \in[0,1] .
$$

Let $\delta$ defined in (3.9) small enough so that $\delta<c_{1}$ and $\gamma$ defined in (3.9) large enough so that $\gamma>c_{2}$. Then from (3.10) and (3.21),

$$
u_{0}(t) \leq v^{*}(t) \leq v_{0}(t)
$$

Note that $T v^{*}=v^{*}$ and $T$ is nondecreasing, we have

$$
u_{n}(t) \leq v^{*}(t) \leq v_{n}(t)
$$

Let $n \rightarrow \infty$ in (3.22), from (3.13) we have that $v^{*}=u^{*}$. Hence, the pseudo-symmetric positive solution to (1.1) is unique.

(ii) From (i), we know that the pseudo-symmetric positive solution to (1.1) is unique. For any $x_{0} \in P$, there exist constants $l_{x_{0}}$ and $L_{x_{0}}$ with $0<l_{x_{0}}<1<L_{x_{0}}$ such that

$$
l_{x_{0}} \omega(t) \leq x_{0}(t) \leq L_{x_{0}} \omega(t) .
$$


Like in (i), we can let $\delta$ and $\gamma$ defined in (3.9) satisfy $\delta<l_{x_{0}}$ and $\gamma>L_{x_{0}}$. Then from (3.10) and (3.23)

$$
u_{0}(t) \leq x_{0}(t) \leq v_{0}(t)
$$

Let $x_{n}=T x_{n-1}$, then

$$
x_{n}(t)= \begin{cases}\int_{0}^{t} \int_{s}^{\frac{1+\eta}{2}} f\left(r, x_{n-1}(r)\right) d r d s, & 0 \leq t \leq \frac{1+\eta}{2}, \\ \int_{0}^{\eta} \int_{s}^{\frac{1+\eta}{2}} f\left(r, x_{n-1}(r)\right) d r d s+\int_{t}^{1} \int_{\frac{1+\eta}{2}}^{s} f\left(r, x_{n-1}(r)\right) d r d s, & \frac{1+\eta}{2} \leq t \leq 1,\end{cases}
$$

where $n=1,2, \ldots$. Note that $T$ is nondecreasing and (3.11), we have

$$
u_{n}(t) \leq x_{n}(t) \leq v_{n}(t)
$$

Let $n \rightarrow \infty$ in (3.25), it follows from (3.13) that $x_{n}$ uniformly converges to the unique pseudo-symmetric positive solution $u^{*}$ for (1.1). At the same time, (3.20) follows from (3.18) and (3.25). Thus, the proof of the theorem is complete.

\section{Example}

Example 4.1. Study the boundary value problem

$$
\left\{\begin{array}{l}
-u^{\prime \prime}(t)=t^{p}(1+\eta-t)^{p} u^{\alpha}(t), t \in(0,1) \\
u(0)=0, u(\eta)=u(1)
\end{array}\right.
$$

where $p \in R, 0<\alpha<1,0<\eta<1$. Let

$$
f(t, u)=t^{p}(1+\eta-t)^{p} u^{\alpha}, \quad(t, u) \in(0,1) \times[0, \infty) .
$$

Note that the function $f$ satisfies that $f(t, u)=f(1+\eta-t, u)$ for $t \in[\eta, 1], f(t, u)$ is nondecreasing in $u$ and if $\sigma \in(0,1]$, there exists constant $\lambda$ with $0<\alpha \leq \lambda<1$ such that $f(t, \sigma u) \geq \sigma^{\lambda} f(t, u)$ for all $(t, u) \in(0,1) \times[0, \infty)$, which coincide with the assumptions (H1) and (H2). Thus, from Theorem 3.1 and Theorem 3.2, we have the following results.

Corollary 4.1. The boundary value problem (4.1) has at least one pseudo-symmetric positive solution if and only if $p>-\alpha$.

Corollary 4.2. Assume $p>-\alpha$. Then

(i) (4.1) has a unique pseudo-symmetric positive solution $u^{*}(t)$, and there exist constants $l$ and $L$ with $0<l<1<L$ such that

$$
l \omega(t) \leq u^{*}(t) \leq L \omega(t), \quad t \in[0,1]
$$

(ii) For any initial value $x_{0} \in P$, there exists a sequence $x_{n}(t)$ which uniformly converges to the unique pseudosymmetric positive solution $u^{*}(t)$ for (4.1), and we have the error estimation

$$
\max _{t \in[0,1]}\left|x_{n}(t)-u^{*}(t)\right|=O\left(1-k^{\lambda^{n}}\right),
$$

where $k$ is a constant with $0<k<1$ and determined by $x_{0}$.

\section{References}

Avery, R. I., \& Henderson, J. (2000). Three symmetric positive solutions for a second-order boundary value problem. Applied Mathematics Letters, 13, 1-7.

Avery, R. I., \& Henderson, J. (2003). Existence of three positive pseudo-symmetric solutions for a one-dimensional p-Laplacian. Journal of Mathematical Analysis and Applications, 277, 395-404.

Çetin, E., \& Topal, F. S. (2012). Symmetric positive solutions of fourth order boundary value problems for an increasing homeomorphism and homomorphism on time-scales. Computers and Mathematics with Applications, 63, 669-678. 
Feng, M. Q., Zhang, X. M., \& Ge, W. G. (2010). Exact number of pseudo-symmetric positive solutions for a $p$-Laplacian three-point boundary value problems and their applications. Journal of Applied Mathematics and Computing, 33, 437-448.

Graef, J. R., \& Kong, L. J. (2008). Necessary and sufficient conditions for the existence of symmetric positive solutions of multi-point boundary value problems. Nonlinear Analysis, 68, 1529-1552.

Guo, Y. P., Han, X. H., \& Chen, Y. R. (2010). Positive pseudo-symmetric solutions for higher order differential equation boundary value problems with sign changing nonlinearities. Communications in Nonlinear Science and Numerical Simulation, 15, 3795-3804.

Hamal, N. A., \& Yoruk, F. (2010). Symmetric positive solutions of fourth order integral BVP for an increasing homeomorphism and homomorphism with sign-changing nonlinearity on time scales. Computers and Mathematics with Applications, 59, 3603-3611.

Ji, D. H. (2008). Triple positive pseudo-symmetric solutions to a four-point boundary value problem with $p$ Laplacian. Applied Mathematics Letters, 21, 268-274.

Jiang, J. Q., Liu, L. S., \& Wu, Y. H. (2013). Symmetric positive solutions to singular system with multi-point coupled boundary conditions. Applied Mathematics and Computation, 220, 536-548.

Luo, Y., \& Luo, Z. G. (2010). Symmetric positive solutions for nonlinear boundary value problems with $\phi$ Laplacian operator. Applied Mathematics Letters, 23, 657-664.

Luo, Y., \& Luo, Z. G. (2012). A necessary and sufficient condition for the existence of symmetric positive solutions of higher-order boundary value problems. Applied Mathematics Letters, 25, 862-868.

Lin, X. L., \& Zhao, Z. Q. (2013). Existence and uniqueness of symmetric positive solution of $2 n$-order nonlinear singular boundary value problems. Applied Mathematics Letters, 26, 692-698.

Ma, D. X., \& Ge, W. G. (2007). Existence and iteration of positive pseudo-symmetric solutions for a three-point second-order $p$-Laplacian BVP. Applied Mathematics Letters, 20, 1244-1249.

Pang, H. H. (2009). Necessary and sufficient conditions for the existence of quasi-symmetric positive solutions of singular boundary value problem. Nonlinear Analysis, 71, 654-665.

Sun, Y. P., \& Zhao, M. (2014). Existence of positive pseudo-symmetric solution for second-order three-point boundary value problems. Journal of Applied Mathematics and Computing. http://dx.doi.org/10.1007/s12190014-0770-9.

Tersenov, Ar. S. (2014). On sufficient conditions for the existence of radially symmetric solutions of the $p$-Laplace equation. Nonlinear Analysis, 95, 362-373.

\section{Copyrights}

Copyright for this article is retained by the author(s), with first publication rights granted to the journal.

This is an open-access article distributed under the terms and conditions of the Creative Commons Attribution license (http://creativecommons.org/licenses/by/3.0/). 